\title{
0318 RISK FACTORS LEADING TO DELIBERATE SELF HARM IN PATIENTS PRESENTING TO THE EMERGENCY DEPARTMENTS: A MULTI-CENTRE CASE-CONTROL STUDY FROM KARACHI, PAKISTAN
}

M Shahid*, A A Hyder, M M Khan, J Razzak, S Jamali, W Kazmi, R Shaikh Correspondence: Aga Khan University, Stadium Road, P0 Box 3500, Karachi, 74800, Pakistan

\subsection{6/ip.2010.029215.318}

Objectives To determine the risk factors of patients presenting with deliberate self harm (DSH) to the emergency departments (EDs) of four tertiary care hospitals of Karachi, Pakistan.

To determine the outcome (admission or discharge) of patients presenting with DSH to the EDs of four tertiary care hospitals of Karachi, Pakistan.

\section{Methods}

Study period March 2010 to May 2010.

Sample Size An equal number of cases (65) and controls (65) will be enrolled from each centre.

Study site Aga Khan University Hospital (AKUH), Abbasi Shaheed Hospital, Civil Hospital Karachi and Jinnah Postgraduate Medical Centre.

\section{Study population}

Inclusion criteria for cases: all patients 18 years and above presenting to the ED of respective hospitals with DSH attempt.

Inclusion criteria for controls: for each case, a control will be enrolled in the study, which will be identified with the use of ED admission register and will be matched with cases for gender and age 2 years.

Exclusion criteria for both cases and controls: individuals not giving informed consent, critically ill (intubated, obtunded patients, those with third degree burns and life threatening injuries).

Study tool DSH-Risk Factors and self-reported questionnaire scale for anxiety and depression.

Psychiatric evaluation, treatment and follow-up The information elicited from questionnaires will help reach a tentative psychiatric diagnosis. These patients will be referred to Psychiatry Consulting Clinics at AKUH. These clinics are conducted by senior psychiatry residents under the supervision of consultants. 